\title{
Understanding Drone Landing on the Human Body
}

\author{
Jonas Auda \\ jonas.auda@uni-due.de \\ University of Duisburg-Essen, paluno \\ Essen, Germany \\ Jessica R. Cauchard \\ jcauchard@bgu.ac.il
}

Magic Lab, Industrial Engineering and Management, Ben

Gurion University of the Negev

Be'er Sheva, Israel

\author{
Martin Weigel \\ martin.weigel@honda-ri.de \\ Honda Research Institute Europe \\ Offenbach am Main, Germany \\ Stefan Schneegass \\ stefan.schneegass@uni-due.de \\ University of Duisburg-Essen, paluno \\ Essen, Germany
}
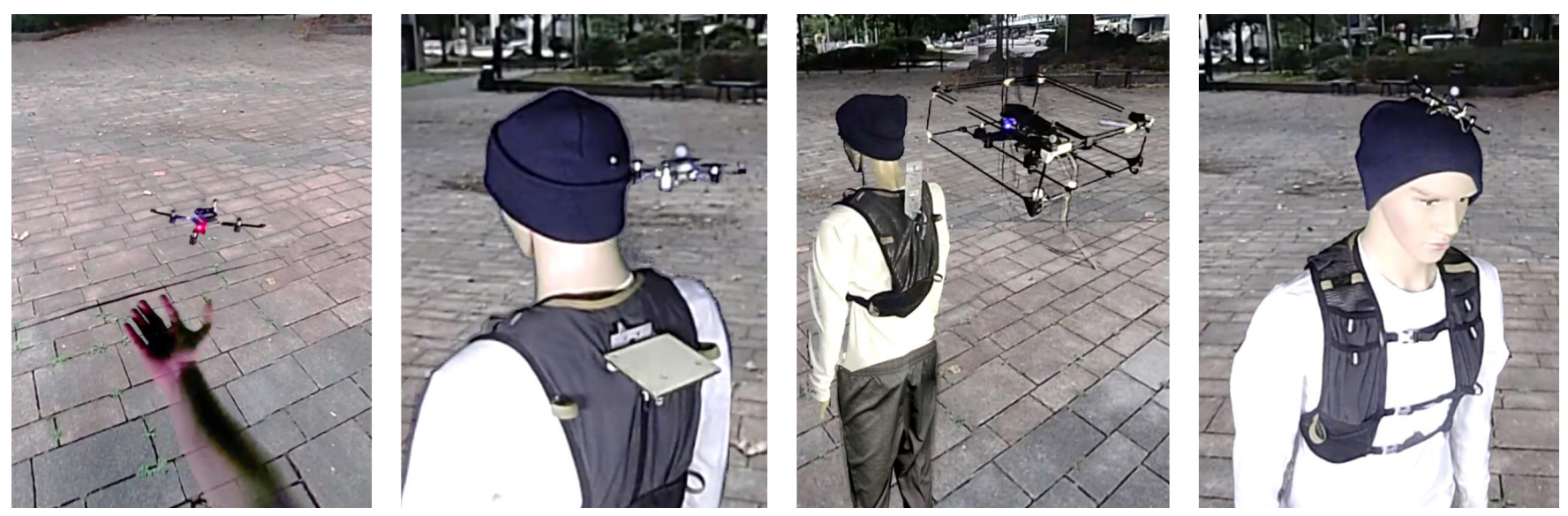

Figure 1: The human body allows for fast and autonomous take-off and landing of drones. We conducted an online survey and a follow-up study with $360^{\circ}$ VR videos to investigate suitability of various body locations (e.g., hand, back, or head).

\begin{abstract}
We envision the human body as a platform for fast take-off and landing of drones in entertainment and professional uses such as medical emergencies, rescue missions, or supporting police units. This new interaction modality challenges our knowledge of humandrone experiences, in which interaction usually occurs at a distance from the body. This work explores important factors for understanding the interplay between drones and humans. We first investigated the suitability of various body locations for landing in an online study $(N=159)$. Our results, presented as body maps, show that the hand and upper back are particularly well-suited body locations. We further tested these findings in a follow-up study $(N=12)$, in which participants experienced drones landing on their bodies through carefully designed and pre-recorded $360^{\circ}$ videos. This immersion into the landing scenarios helped us to identify common themes and research approaches for different body parts. Taken
\end{abstract}

This work is licensed under a Creative Commons Attribution International 4.0 License.

MobileHCI '21, September 27-October 1, 2021, Toulouse \& Virtual, France (c) 2021 Copyright held by the owner/author(s).

ACM ISBN 978-1-4503-8328-8/21/09.

https://doi.org/10.1145/3447526.3472031 together, the findings provide first insights into location preferences and themes for drones landing on the human body.

\section{CCS CONCEPTS}

- Human-centered computing $\rightarrow$ Human computer interaction (HCI); User studies; Virtual reality; • Computer systems organization $\rightarrow$ Robotics.

\section{KEYWORDS}

Drone; UAV; Micro Air Vehicle; On-Body Landing; Mechanical Turk; Virtual Reality Study; Human-Drone Interaction

\section{ACM Reference Format:}

Jonas Auda, Martin Weigel, Jessica R. Cauchard, and Stefan Schneegass. 2021. Understanding Drone Landing on the Human Body. In 23rd International Conference on Mobile Human-Computer Interaction (MobileHCI '21), September 27-October 1, 2021, Toulouse \& Virtual, France. ACM, New York, NY, USA, 13 pages. https://doi.org/10.1145/3447526.3472031

\section{INTRODUCTION}

In recent years, it has become common for drones to solve tasks that are outside of the reach of the human (e.g., conducting inspection at large heights). We can expect that the interaction between autonomous flying drones and humans will further increase over 
time. Drones may be used in a wide range of scenarios, such as controlling crowds in an emergency [41], delivering urgently needed medicine to a patient [50], assisting search an rescue missions by providing an overview [18, 31], for work or sports [29, 34] or even entertainment [40,42], or to enhance virtual experiences [1,22, 26]. Further, drones can assist their owners in various tasks, for example, providing navigation aid [4, 27]. They are also widely adopted for photography, filming, or delivery [47].

Drones usually keep their distance from the user and land in the vicinity before they are manually picked up and stored away in boxes. However, this makes their usage cumbersome and limits their use to situations with free hands and no time constraints. In this research, we envision the human body as a base station for one or multiple drones to enable fast landing and take off.

In Human-Drone Interaction (HDI) research, four major fields have emerged - Control Modalities, Human-Drone Communication, Proxemics, and Novel Use-Cases [48]. However, the HDI community lacks an understanding about the user's common themes, as well as, if and where users would accept drones landing on their body [3]. This is important, since the body is a personal space. People may have different opinions about what constitutes an appropriate interaction between drones and their bodies. Further, investigating how a drone must be visually designed to land on the human body is important, as certain designs could negatively influence the perception of a drone [9].

In this paper, we address these issues by reporting the findings of two user studies. In our findings we identify common themes and investigate the acceptability of autonomous drone landing on the human body. In the first study, we conducted an Amazon Mechanical Turk (MTurk) survey with 159 participants to understand the preferred landing location and opinions on the visual appearance of drones that land on the body. We contribute body maps showing the acceptability of drone landing during four activities: standing, walking, sitting, and climbing. These body maps can help researchers and practitioners to find well-accepted locations for drone landing. Based on our findings from the survey, we implemented a software framework to enable autonomous drone landing on the human body. In particular, we investigated landing on the hand, back, shoulder, and head by building various body-worn landing mechanisms. The software framework supports two drones of different sizes and eases the specification of custom landing maneuvers.

We used the software framework to conduct a second study with 12 participants. We aimed to deepen our understanding by investigating six drone-landing maneuvers in $360^{\circ}$ videos on virtual reality headsets. The videos were created using two autonomous drones that landed on a mannequin. The immersion into the VR landing scenario revealed common themes, metaphors, and preferred approach behaviors for drones landing on the human body. It demonstrated that preferred landing locations are the hand and back, drones should indicate landing intentions, and approach the user in a controlled and precise trajectory. Taken together, our results demonstrate a great potential for autonomous drone landing on the human body.
In summary, this paper contains three main contributions:

(1) Findings from an MTurk survey $(N=159)$ on the suitability of body locations for drone landings, visual appearance of drones landing on the body, and users' common themes. We contribute body maps that visualize location preferences in four activities and we derive common themes, including safety, comfort, and visibility.

(2) An implementation of different drone landing maneuvers using two drones. We made this software framework opensource to accelerate and ease the development of new drone landing maneuvers.

(3) Insights from a VR user study $(N=12)$ in which participants were immersed into six landing scenarios. We contribute results from a questionnaire and a semi-structured interview. We found that the hand and back are particularly well-suited for drone landing. We also obtained suggestions about how drones should carry out landing maneuvers.

\section{ENVISIONED APPLICATION SCENARIOS}

In many scenarios, a drone operator might not have the time, mental focus, or physical space for landing and storing a drone. For instance, rescue personnel, paramedics, and police officers might need to get an overview of their environment multiple times during a mission. However, they are too occupied with safety-critical tasks and the terrain might not always be suitable for traditional drone landings; for example, while climbing a mountain or standing in a crowded space. Autonomous drones could return after completing their task and automatically land on a suitable body location, without restricting the operator's movements and without requiring human intervention. In other scenarios, the drone might land on people, who are not the owner or operator of the drone. For example, autonomous or teleoperated medical drones could land on people to perform basic first-aid and vital monitoring to support triage after mass casualty events.

Finally, our vision extends prior work on nonaerial on-body robots [11]. Drones could interact with the human body while landed, e.g., by giving tactile feedback. Additionally, they can perform tasks beyond the human body while in the air. We believe this will lead to more social drones in the future that are able to support people during their daily lives [7].

\section{RELATED WORK}

This work relates to the domains of collocated Human-Drone Interaction (HDI) and proxemic interactions and takes inspiration from prior VR study methodologies.

\subsection{Collocated Human-Drone Interaction}

Small-sized drones are increasingly present in human environments, used for both leisure and in professional settings. They are already helping people in a plethora of applications from journalism and agriculture, to surveying, scientific work, and even search and rescue [44]. As drones become increasingly autonomous, low level remote control becomes redundant and collocated interactions prevail [6]. Researchers have proposed different mechanisms for collocated HDI, such as using hand and body gestures $[6,15,16,33,36-38]$ or gaze [25]. Recently, researchers have investigated the use of touch 
$[2,5,30]$ where the drone can be interacted with at arm's reach. For example, Lieser et al. let a drone fly specific dance trajectories to electronic music after detecting being touched recurrently on its frame by a user [30]. These trajectories depend on the position of touch on the frame. The intention was to provide a playful bond between human and drone. Such interaction might become more prominent in the future thanks to new form and shapes of drones that are now safe to touch [21]. This enables new paradigms of interaction where drones could come all the way to the person's body. Such metaphors have been proposed as potential natural interaction techniques, where drones could land on the forearm, using a falconry metaphor [37]. Commercial products have now followed suit, giving the ability for a drone to land on a person's hand [43]. Inspired by $\mathrm{Ng}$ and Sharlin [37], we propose that the body could become a platform for the drones to land and take off from. Yet, much is unknown about how a drone should approach a person and what body locations are suitable to land and take off.

\subsection{Proxemics}

Hall introduced the notion of proxemics [19] as a way to organize interaction spaces and distances in people to people communication. It is divided in four zones, from intimate to personal, social, and public. Much research has explored the notion of proxemics in robotics $[32,35,46]$, showing how different mechanisms around the design and behavior of robots can mitigate the acceptability of human-robot interaction within different interaction spaces. In HDI, several works have investigated proxemics $[12,51,52]$. Recently, Wojciechowska et al. [51] showed that people prefer for a drone to stop within their personal space and that when the drone enters the intimate space, people's comfort levels decrease. This is on a par with prior work in ground robotics [23], although it was found that people are comfortable with flying robots getting closer to them than ground robots [49]. These prior works confirm that getting the drone from the personal to the intimate zone is not straightforward. Since prior works [12, 51, 52] investigated how different factors, including drone shape, speed, movement, position, and approach strategies influenced human preferences - from the public to the personal spaces. We propose to take the research one step further and investigate the acceptability of drones landing on the body, i.e., entering the intimate space. Our work focuses on both uncovering suitable body locations for drones to land and take off and identifying characteristics for acceptability. The next section presents research methodologies related to our approach that foster safety while preserving certain aspects of validity.

\subsection{HDI Research Methodologies Using Simulations in VR}

Simulating drones in virtual or augmented reality provides medium realism and complexity while reducing the safety risk, according to Wojciechowska et al. [51]. In fact, here VR studies achieve second best realism with real co-located flights achieving the highest realism. Furthermore, using VR or AR to simulate drones fosters reproducibility. Different approaches were employed in prior research to study users' perception of a drone and how to communicate the drone's intentions or behavior. To study users' perception and attitude towards a drone companion in a home environment Karjalainen et al. simulated a virtual home in VR [24]. Prior to the VR study they designed a drone based on results from questionnaires and workshops. The results from the VR study indicated that the virtual drone matched the expectations of the participants in the context of a home environment. Duncan and Murphy studied HDI using a 2D-CAVE virtual environment [13]. They simulated drones were flying at different speeds or with different cyclic flight motion. The results indicate that low flight speed and cyclic flight motion resulted in larger distances between the human and the drone. Both approaches used virtual simulations to research HDI. Common to these approaches is a low safety risk that is of utmost importance in the field. We opted for a similar approach: bringing our drones in VR through pre-recorded $360^{\circ}$ videos that can be viewed on an immersive VR-HMD.

\section{RESEARCH QUESTIONS}

The body is a new landing domain for drones, which raises a lot of new questions for Human-Drone Interaction research. In the following, we specify the four main research questions that guide our study design:

\section{RQ1: Which parts of the human body are suitable landing locations for drones?}

We study the user acceptance of drones landing on various body locations. While there might be technical limitations in future drones, in this work we are primarily interested in common themes and user preferences. We evaluate this research question with the quantitative results of our online survey, as well as, the qualitative feedback on the different landing locations from our VR study.

\section{RQ2: How is landing acceptability influenced by drone design?}

While the design of today's drones is primarily technology-driven, there is already a wide variety of visual designs [50]. Since we imagine that drones landing on the body can create stronger bonds between people and their drones, we believe visual aesthetic and fashion might play a stronger role in the future. We evaluate this by having participants rate 25 drone designs in the online survey.

\section{RQ3: How do activity and context influence drone landing acceptability?}

We believe activities and context play an important role in the acceptability of drone landings because the activity determines the movement and orientation of body parts. In the MTurk study, we evaluate four activities with different intensities: sitting, standing, walking, and climbing. Moreover, we asked participants in the online survey and VR study in which context, use-cases, and situations they find the landing of drones acceptable.

\section{RQ4: What influence do flight path and landing behavior have when landing on the human body?}

Drones can follow many different flight patterns. This does not only have a functional purpose, but also helps in the communication 
Table 1: Previous drone experience \& technology interest of the 159 participants on a 7-Point-Likert-Scale (1: Strongly disagree - 7: Strongly agree).

\begin{tabular}{lccc}
\hline Question & Mean & SD & IQR \\
\hline I have experience with drones or quadcopters & 4.75 & 1.95 & 2.0 \\
I am interested in new technology & 5.84 & 1.38 & 2.0 \\
I am using new technology regularly & 5.48 & 1.48 & 1.0 \\
I would consider myself tech-savvy & 5.26 & 1.64 & 1.0 \\
\hline
\end{tabular}

between drone and human (e.g., by expressing emotions [8]). We investigate different flight paths and landing behaviors in our VR user study by asking participants for their opinions on six different landing approaches.

\section{ONLINE SURVEY}

We conducted an MTurk online survey to understand location preferences and common themes of 159 participants.

\subsection{Study Procedure \& Participants}

We conducted an online survey using the MTurk platform to crowdsource feedback on suitable spots on the human body for a drone to land. Crowd-sourcing platforms like MTurk are applied across various research domains, for example, wearables to obtain a reasonable representation for the population [20]. In total, 210 participants took part in our survey. We excluded 51 participants who did not answer a control question properly in order to eliminate participants that quickly skipped through our survey. In total, 159 participants (100 male, 57 female, 1 non-binary, 1 unspecified gender) completed the survey successfully. The participants reported an average age of 33.68 years $(S D=10.42, I Q R=9.0)$. In terms of education, $93.01 \%$ of the participants had a Bachelor's degree or above.

We asked the participants how often they had used drones in the past. Fifty-three (33.33\%) reported having used drones 1-2 times, 41 (25.79\%) reported 3-5 times, 12 (7.55\%) reported 5-10 times, and 11 $(6.92 \%)$ reported more than 10 times. A total of $19(11.95 \%)$ participants had never used drones. Of those with experience, 88 (55.3\%) participants reported that they had piloted a drone by themselves. We also asked the participants about what kind of drone experience they had. A total of 68 (42.77\%) reported that they had used drones in the context of photography or filming, such as at a wedding or for video shoots. A total of 32 (20.13\%) participants reported flying drones as a hobby. Twelve $(7.55 \%)$ participants stated that they had conducted drone races. Two $(1.26 \%)$ participants reported that they had stood by while another person flew drones. One $(0.63 \%)$ participant was building a drone for aerial security. The remaining participants did not specify any particular usage. Further subjective ratings regarding drone experience and technology interest can be seen in Table 1.

\subsection{Acceptance of On-Body Landing Locations}

One major research question we addressed in the online survey was the acceptability of different landing locations on the body (RQ1). We asked participants to mark various body parts for one of four activities (RQ3) as either acceptable or inacceptable for a drone to land. Based on this data, we rendered the heat maps in Figure 2. Further, we calculated acceptability percentages across all body maps (see Table 2). We have chosen activities such as standing, walking, and sitting as they represent everyday activities. In addition, we picked climbing as a demanding mobile activity, which is common in search and rescue missions. In the climbing scenario, the drone operator has occupied hands, is located in extreme terrain, and is focused on another task.

5.2.1 Always Acceptable Landing Locations. The hands, the shoulders, and the back and front of the arms were rated as most acceptable across all body maps. On average, the hands have the highest acceptability of $78.10 \%$. This is followed by the shoulders with $77.29 \%$ and the arms with $74.06 \%$. Next, the upper torso was rated as acceptable for drone landing with a percentage of $66.87 \%$. The feet were rated $62.33 \%$, followed by the lower torso $(61.72 \%)$. The legs received a percentage of $61.05 \%$. Overall, the head was rated with the lowest acceptability $(54.91 \%)$.

5.2.2 Activity Differences. We compared the acceptance of landing on the front of the participant in three activities: sitting, standing, and walking (see Table 3). We excluded climbing from our analysis, since the body is pressed towards a wall and does not allow for drone landings from the front. Our findings show that participants found, on average, more landing locations acceptable while standing (67.0\%). This was followed by walking $(65.0 \%)$ and sitting $(62.87 \%)$. It is particularly visible when comparing the lower body of sitting, standing, and walking that the activity influences acceptance. Although participants found the legs and feet to be acceptable landing locations while inactive (e.g., sitting and standing), they were considered to be unacceptable during movement (e.g., walking). In addition, joint areas such as the knees were rated as less acceptable than were flat areas such as the upper or lower legs.

\subsection{Rating of Drone Design as a Factor for Landing Suitability}

The design of drones could be a major factor in the suitability of onbody landing of drones (RQ2). We selected 25 drones (see Figure 3) that are commercially available from prior work on drone design [50]. We selected drones from different categories (e.g., appearing pet-like, machine-like, intelligent, or mature) based on the classification of Wojciechowska et al. [50]. We asked our participants to rate whether the drone looks suitable for landing on the human body on a 7-Point Likert Scale (1: Strongly disagree - 7: Strongly agree). We sorted the drones in Figure 3 according to their received rating for landing suitability. Afterward, we asked the participants to specify the reasoning behind their ratings for the landing acceptance and suitability of the 25 presented drones.

5.3.1 Shape, Size \& Weight. The participants explicitly mentioned that the shape of the drone did influence their decision-making. The participants were concerned about spiky parts such as rotors or "legs". Furthermore, size and weight were listed as limiting factors of the landing suitability. The participants mentioned that flat drones seem more suitable for landing on the human body. For example, the "legs" of the drones were mentioned frequently (12 times). The participants stated that "spiky parts" and "pointy legs" seem unsuitable for landing and could pose an injury risk. 


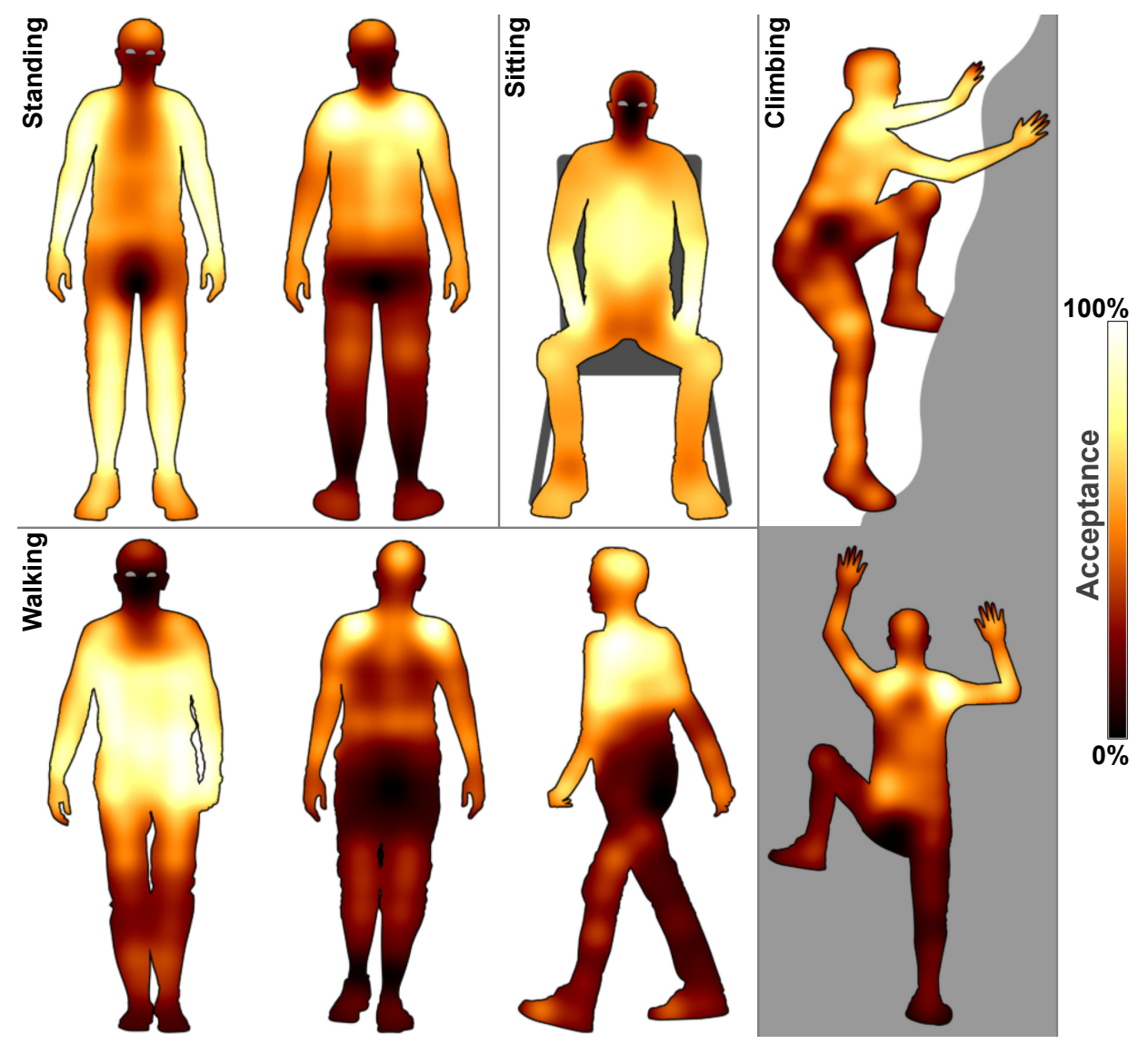

Figure 2: Location preferences for drone landings on the human body while standing, sitting, walking, and climbing.

Table 2: Drone landing acceptability ratings for different body locations.

\begin{tabular}{llllll}
\hline Body Location & Mean & SD & Med & Min & Max \\
\hline Head & $54.91 \%$ & $16.3 \%$ & $59.02 \%$ & $15.69 \%$ & $69.44 \%$ \\
Shoulders & $77.29 \%$ & $13.38 \%$ & $80.0 \%$ & $50.0 \%$ & $100.0 \%$ \\
\hline Arms & $74.06 \%$ & $7.98 \%$ & $72.7 \%$ & $60.5 \%$ & $85.6 \%$ \\
Hands & $78.1 \%$ & $7.42 \%$ & $77.21 \%$ & $66.67 \%$ & $88.89 \%$ \\
\hline Upper Torso (front \& back) & $66.87 \%$ & $7.11 \%$ & $66.76 \%$ & $57.01 \%$ & $77.31 \%$ \\
Lower Torso (front \& back) & $61.72 \%$ & $9.31 \%$ & $62.92 \%$ & $50.0 \%$ & $79.44 \%$ \\
\hline Legs & $61.05 \%$ & $9.99 \%$ & $60.73 \%$ & $46.67 \%$ & $75.2 \%$ \\
Feet & $62.33 \%$ & $6.48 \%$ & $59.8 \%$ & $55.0 \%$ & $72.22 \%$ \\
\hline
\end{tabular}

5.3.2 Drone Design. The design of the drones further influenced the landing acceptability. Some reasons for unsuitability were a militaristic appearance or an insect or spider-like character. A more friendly appearance was required for landing suitability. The color of the drone was also mentioned by the participants, as a dark or black drone was perceived negatively: "I can let the more colorful drones land on me, as they seem a bit more friendly, and smaller[...]".
5.3.3 Risk of Injury. As an important decision factor, risk of injury was mentioned explicitly by 18 participants. Sharp parts were considered to make a drone unsuitable for landing. On the one hand, larger size was believed to increase the risk of injury: "[...] some look to be the size of my body, that could crush me and kill me". On the other hand, participants welcomed safety features such as a "strong frame" and "blade guards" to protect the skin.

5.3.4 Use Cases. The participants mentioned that the use case influenced their decision-making. For example, they stated it could 


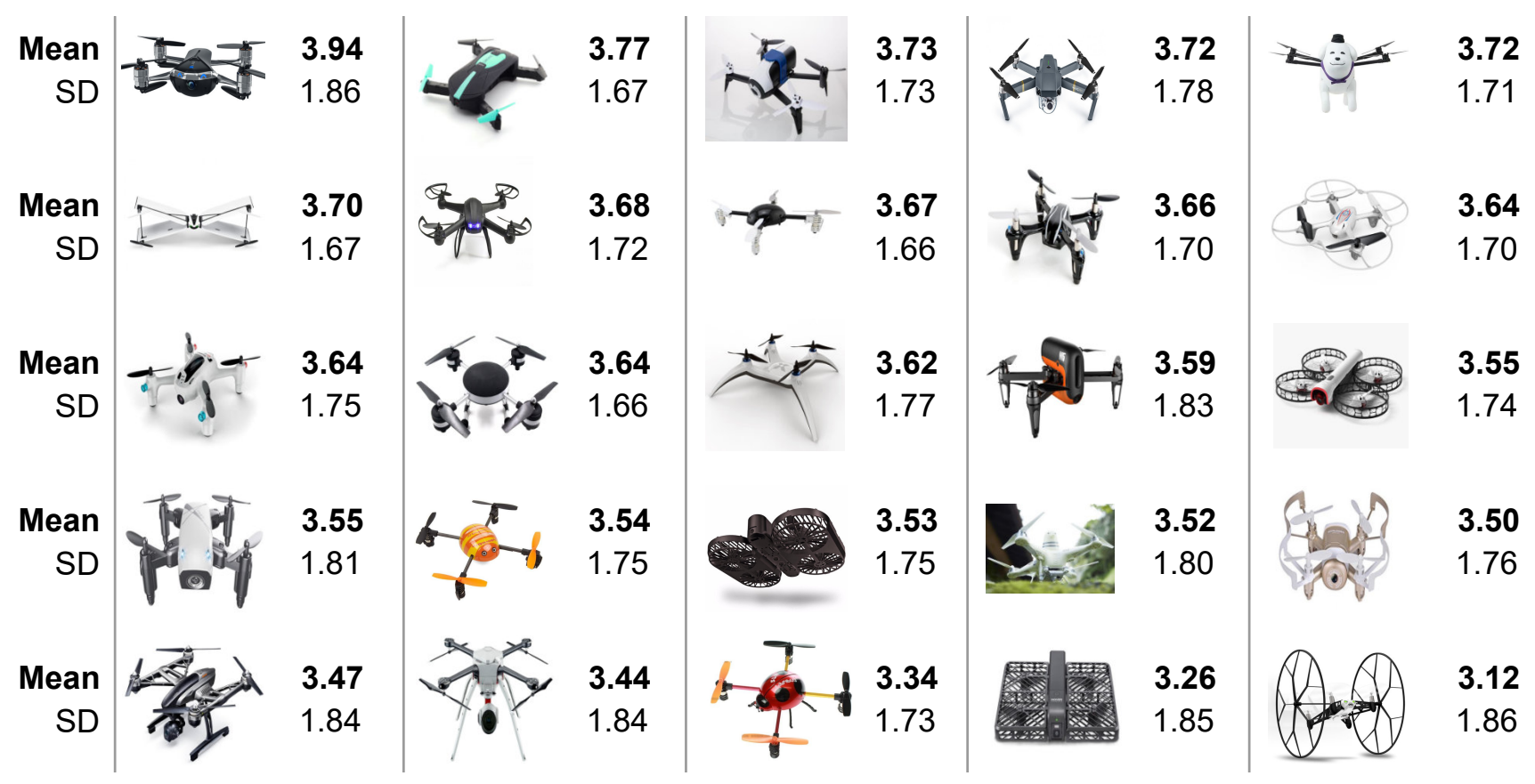

Figure 3: Drones with various designs and their average suitability ratings for landing on the human body (7-point Likert scale).

Table 3: Drone landing acceptability ratings for different activities.

\begin{tabular}{llllll}
\hline Activity & Mean & SD & Med & Min & Max \\
\hline Standing & $67.0 \%$ & $8.56 \%$ & $64.5 \%$ & $53.0 \%$ & $81.0 \%$ \\
Walking & $65.0 \%$ & $9.43 \%$ & $64.0 \%$ & $51.0 \%$ & $79.0 \%$ \\
Sitting & $62.87 \%$ & $9.66 \%$ & $65.0 \%$ & $46.0 \%$ & $77.0 \%$ \\
\hline
\end{tabular}

be acceptable for a drone to land on a human in the case of a medical emergency or in "[...] risky situations such as natural disasters".

\subsection{Acceptable Use Cases and Situations for Drone Landing}

We asked the participants to rate the acceptance of landing a drone on their bodies for different use-cases and situations (RQ3) on a 7-point Likert scale.

5.4.1 Use-Cases. The acceptance was rated highest for rescue purposes with an average of $4.43(\mathrm{SD}=1.37, \mathrm{Med}=5.0, \mathrm{IQR}=1.5)$ followed by work-related purposes $4.28(S D=1.49$, Med $=5.0, I Q R=2.0)$ and medial emergencies with $4.02(S D=1.65$, Med $=4.0, I Q R=$ 2.0). Landing a drone for entertainment purposes were rated the least with $3.89(S D=1.62, \mathrm{Med}=4.0, I Q R=2.0)$.

A Wilcoxon Signed-Ranks test (Bonferroni corrected) indicated that landing drones for rescue purposes was rated more acceptable than for entertainment purposes, $(W=3269.50, p=.027)$. Same for work purposes compared to entertainment purposes $(W=$ $2502.00, p=.048$ ) and for rescue purposes compared to medical emergencies $(W=1389.5, p=.017)$. Other comparisons did not reveal significant differences.

5.4.2 Situations. The acceptance for landing a drone on the body while being indoors was rated lowest with an average of $3.82(S D=$ $1.68, \mathrm{Med}=4.0, I Q R=2.0$ ) followed by landing while working with $3.99(S D=1.66$, Med $=4.0, I Q R=2.0)$ and while doing sports 4.04 $(S D=1.75$, Med $=5.0, I Q R=2.0)$. During free time received an average rating of $4.14(S D=1.63$, Med $=5.0, I Q R=2.0)$. Last, being outdoors was rated highest with $4.5(S D=1.41, \mathrm{Med}=5.0, I Q R=$ 1.0). Wilcoxon Signed-Ranks test indicated that landing drones for while being outdoors was rated more acceptable than while being indoors $W=2686.00, p=.001$, more acceptable than during sport $(W=1467.00, p=.017)$, and while working $(W=1836.50, p=.007)$. Other comparisons did not reveal significant differences.

\subsection{Themes}

We extracted common themes from the answers of the open-ended questions in our MTurk study. Four authors used thematic analysis [10] for the qualitative analysis of this set of data. We coded the free text answers from the MTurk study simultaneously by moving and annotating the data on a collaborative whiteboard. From the created clusters, they extracted themes related to the acceptability of landing on different body locations. The whole process was conducted in two sessions and concluded once all researchers agreed on the themes and coding of the data, which took a total of 12 person-hours We found six different categories, the participants used for reasoning about the acceptability of landing on different body locations: 
5.5.1 Safety. Most statements were related to safety. Participants found places unacceptable that have "too much risk of getting severely hurt" and mostly accepted places "where it would not possibly hurt me". Many participants found it "unsafe to land a drone near the head or face because of the risk of injury from the drone". Some were concerned with locations with "exposed skin or sensitive pain areas", which "might not heal fast and can bleed a lot if the fans cut a person", possible "damage to vital organs", and that "the fan might twist the hair". They preferred "[...] healthy parts which are quite strong[...]" and "area will be easy to control if at all a damage it about to occur". One participant positively mentioned "legs, knees and feet as good places to land because those are places that can take some impact during sports like soccer."

5.5.2 Comfort \& Appropriateness. Participants mentioned comfort as another important factor for their ratings and found places unacceptable that "cause immediate discomfort". For example, one participant states "any bony areas such as elbows and ankles would not make for a comfortable landing”. Multiple participants mentioned they prefer less sensitive body locations for drone landing, because "the body doesn't get affected as much". Another participant found body locations unacceptable, "because these areas are very sensitive and not comfortable to land drones". Beyond physical comfort, participant were concerned about the appropriateness of the landing location. One participants found it "inappropriate [...] to land a drone on the butt, sides, stomach, tights and face". Another participant mentioned "landing on my bum will be offensive to me" and another mentioned "the hip could also be used as a landing spot even though it's sort of awkward".

5.5.3 Convenience \& Restrictions. Convenience was mentioned as another important factor. For example, landing locations should be "easily accessible". Drones on some body parts might be inconvenient, since they impair the balance of the body. Some mentioned "the legs are not suitable for the drone to land on, because the body rested on the leg". Further, drones should not restrict the movement of the body. Participants mentioned they prefer locations that "won't affect the person" and where a drone is "not in the immediate way of action". These restrictions not only include movements, but also other factors like the sight of the person. Participants were also concerned that this would require a certain fitness level and that specific "areas are moving too much [and] too quickly". One participant mentioned that especially while climbing, "adding another element makes the risk higher of losing grip and falling to [...] death".

5.5.4 Visibility. Participants mentioned they would like to be able to see the drone and the landing area. They mentioned that landing on locations with bad visibility (e.g., the back) might be "too much of a surprise" and people might "get panic when the drone suddenly lands". This is also reflected in the heat maps in Figure 2: Locations in the front of the person have on average a higher acceptability compared to locations with limited visibility behind them.

5.5.5 Drone Capabilities. Although we did not mention any specific landing capabilities of drones during the online survey, many participants mentioned technical considerations as a key factor for their acceptance rating. They accepted landing on body parts that are "flat" and "horizontal", as well as, "remain stable even while walking". They also preferred larger spaces to "ease the landing" and locations that can "withstand the drones weight". They found body parts that are "unstable", "shake", or have "too much movement" unsuitable for landing.

Participants preferred "easy places where the drone can land". This includes "top surfaces" (e.g., "the top of the head is alright for landing as it is easy for the drone to just sit on the head"), "small places" (e.g., "landing on the feet or other small body parts would be difficult"), and "smooth area[s] to [...] not fall off".

5.5.6 Interaction \& Control. Some participants mentioned they prefer hands and arms, since these locations gives them the possibility to interact with the drone and a high level of control. People can "convenient hold the drone", "easily catch the drones", "easily grab it, control it", "convenient to take off the body", and "escape from any accident".

\section{VIRTUAL ENVIRONMENT STUDY}

To get a deeper understanding of the insights from the online survey and to further investigate suitable body location, we developed a software framework to precisely steer drones to a specific location on the human body. We incorporated two differently sized drones into the system - a small drone and a larger drone. We prerecorded $\operatorname{six} 360^{\circ} \mathrm{VR}$ videos of the two drones. These videos included landing on various body parts i.e. the back, head, arm, and the shoulder. We then showed these videos to the participants using an VR HMD together with stereo headphones and gathered qualitative feedback through questionnaires and semi-structured interviews. In the following, we describe the technical implementation of the software framework we used to record the $360^{\circ}$ VR videos.

\subsection{Drone Control Software Framework}

Our software framework consist of two drones, a control\&command application, and an optical tracking component.

To precisely track the position and orientation of our drones in 3D-space, we used an OptiTrack $13 \mathrm{~W}$ optical tracking system. The spatial data from the OptiTrack system was streamed to a control application in real-time. For the system to see the drones, we equipped them with optical tracking markers. The control server determined yaw, pitch, roll and throttle for the drone to reach a specific position. To precisely steer the drones, we tuned PID controllers to the given physical properties of the drones until we obtained flight trajectories that diverge by less than $\sim 3 \mathrm{~cm}$ from the specified position. The commands were send by radio to the drones. The code of the complete system is available under MIT license on GitHub ${ }^{1}$. We integrated two drones in our system, which we introduce in the following:

6.1.1 Small Drone. As a smaller drone we used an off-the-self CrazyFlie 2.0 $0^{2}$. It measures $9.2 \times 9.2 \times 2.9 \mathrm{~cm}$ with an weight of $27 \mathrm{~g}$. To control the CrazyFlie, we used a Crazyradio PA USB radio dongle attached to computer running the control application.

6.1.2 Large Drone. We build a custom F3 Flight drone measuring $35 \times 38.5 \times 14.5 \mathrm{~cm}$ with an weight of $750 \mathrm{~g}$. We used a F3 Flight Controller Acro 6 DOF as the central control unit of the drone. As a frame we used a QAV250 250mm Carbon Fiber Quadcopter Frame.

\footnotetext{
${ }^{1}$ https://github.com/jonasauda/understanding_drone_landing

${ }^{2}$ https://www.bitcraze.io/products/crazyflie-2-1/
} 

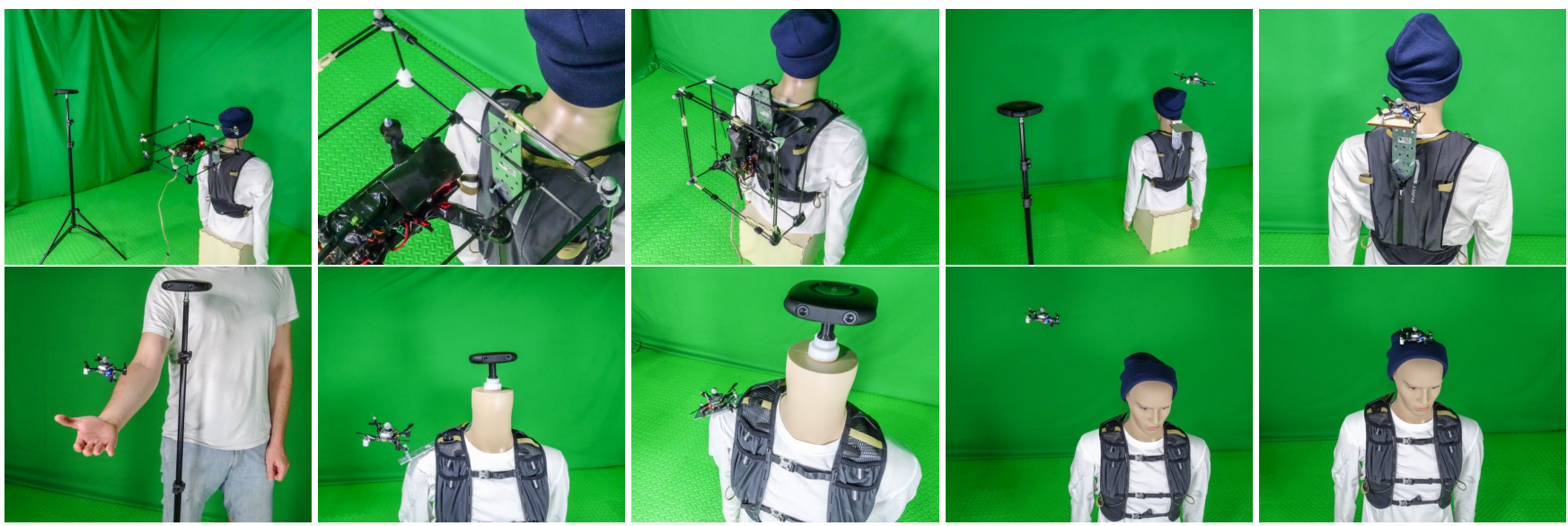

Figure 4: We recorded six drone maneuvers with a $360^{\circ}$ camera. The camera was either mounted on a mannequin to record 1 st person view videos or on a tripod to record from a 3rd person view.

We mounted four motors (MT2204 $2300 \mathrm{KV}$ ) controlled by four ESCs (Emax 12A Simonk ESC) to the frame. Further, we mounted 5030 propellers on the motors. We soldered together all the wires between the power distribution board and the components. To receive the radio signal, we added a NodeMCU to the drone equipped with an WayinTop 2pcs NRF24L01+PA+LNA transceiver. The received signal was forwarded via PPM from the NodeMCU to the flight controller. We attached a Keywish RF-Nano Arduino Nano V3.0 to the computer running the control application via USB to radio control commands to the drone. For safety, we build a cage out of carbon sticks ${ }^{3}$ that surrounds the drone to keep distance between the rotors and humans or obstacles.

6.1.3 Platforms. We built three landing platforms to ease the drone landing and to analyze the impact of the landing platform on the acceptability of the user. To land a drone on the shoulder, we used a metal plate with hooks (to prevent a landed drone from falling off) that is attached on one of the user's shoulder (cf., Figure 4). The metal plate juts over the shoulder of the wearing user. For landing a small drone on the back, we attached a horizontal plate to the backpack measuring $10 \times 10 \mathrm{~cm}$. To land a large drone, we attached a vertical plate with hooks to the back pack. The drone could use its cage to attach itself to the hooks and would then hinge itself down to be worn like a back pack by the user.

\section{$6.2360^{\circ}$ Recordings of Landing Maneuvers}

We used a Vuze 3D $3604 K$ VR Camera to record our drones converging on different body location. Prior to recording, we covered the walls, floor, and ceiling of our lab with green screens to digitally add another background video in post production. Therefore, we recorded a video of an urban setting (i.e., an open square with buildings, trees, and cars in the background). As we could not cover the cameras of the tracking system because it was needed to steer the drones, we digitally masked these areas in the $360^{\circ}$ video in order to properly edit the cameras our of the video. We recorded seven scenes - six flight maneuvers and one video to familiarize

\footnotetext{
${ }^{3}$ http://www.hirundino.com/beyond-the-force/do-it-yourself
}

Table 4: The average technical affinity of the participants on a 7-point Likert scale.

\begin{tabular}{lccc}
\hline Question & Mean & SD & IQR \\
\hline I have experience with drones or quadcopters & 2.25 & 1.83 & 1.75 \\
I am interested in new technology & 5.25 & 1.53 & 1.0 \\
I am using new technology regularly & 4.83 & 0.9 & 1.25 \\
I would consider myself tech-savvy & 4.83 & 1.82 & 2.0 \\
\hline
\end{tabular}

with the situation of having a drone flying in proximity. To avoid injuries while filming, we used a mannequin as a model for the human body (cf., Figure 4). Instead of the head, we mounted the camera to the mannequin to record 1st person view videos. For landing the users hand, an actor reached out with the arm for the drone to land on. Landing on the head and back was filmed from a 3rd person view because otherwise one could not see the drone approaching or landing. For landing on the shoulder, we attached a platform on the mannequins right shoulder.

We excluded climbing and other similar sports activities from our VR study since they did not fit the urban context. We elaborate on such activities and further settings for drone landing on the human body in the future work.

\subsection{Participants}

We recruited 12 participants ( 6 female, 6 male, ten right-handed, two left-handed) with an average age of 30.92 years $(S D=12.41, I Q R=$ 9.75). We asked the participants what kind of drone experience they have. They reported that they have used drones for fun, filming, or education (programming). One participant reported an encounter with remotely controlled drones in real life. In this situation the participant wanted to pass by a specific area. Because of a possible crash with the drones that was not possible. From the 12 participants, 7 reported that they have never used a drone in the past. Three participants used drones for 1-2 times before. One participants for 3-5 times and one more than 10 times. From the 12 participants 4 reported that they have piloted a drone by themselves. Further, 


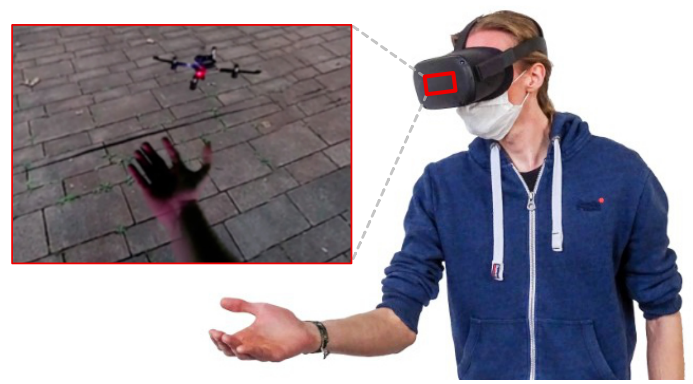

Figure 5: A participant experiences a drone landing on the hand in a $360^{\circ}$ video.

we asked the participants if they had previous Virtual Reality (VR) experience. Seven out of 12 participants reported that they have used VR once before. Two said they use VR on a monthly basis and two have never used VR before. Finally, we assessed the technical affinity of our participants on a 7-Point-Likert scale (see Table 4).

\subsection{Apparatus \& Procedure}

We started the study by showing each participant a video in VR to familiarize with the setting. Therefore, we handed the participants a head-mounted display (Oculus Quest) with attached stereo headphones (beyerdynamics Custom One Pro). In this video, our small drone (CrazyFlie) flew repeatedly in front of the user. After the participant reported to have acclimated to the setting, we started with the first landing video. The landing videos were counter balanced (Latin Square Design). In total, each participant watched six landing videos (cf., Figure 5). The participants could watch each video multiple times. After each video, we asked the participants to rate nine statements on a 7-Point-Likert scale. After each rating, we asked for reasons why a specific value has been picked. Then we proceeded with the next video. We instructed the participants to stand still while watching the $360^{\circ}$ videos or hold their hands as seen in the video in which the small drone was landing on the viewers hand (cf., Figure 5). Further, as we recorded landing on the head and back from the 3rd person view, we instructed the participants to empathize with the mannequin on which the drones landed. Otherwise, the participants would not recognize the drone because they would not be able to see it nor feel it due to missing haptic feedback in current VR systems. After each participant has seen all six landing videos, we conducted a semi-structured concluding interview about the virtual drone experience.

\subsection{Results}

We asked each participant to rate their virtual drone experience with regards to drone flight behavior, visual appearance, auditory appearance, and experienced safeness. In total, the participants rated nine statements (S1 - S9) on a 7-Point-Likert scale (cf., Figure 6). The exact results for the small and large drone can be obtained from Table 7 and Table 8 respectively in the supplementary materials. Wilcoxon Signed-Ranks (Bonferroni corrected) indicate significant differences regarding the different body parts used for drone landing (see Table 6 in the supplementary materials). In the following, we introduce the feedback from our participants gathered in a semi-structured interview. We introduce them clustered into themes:

6.5.1 Landing Platforms. We used the landing platform and hook primarily to ease the landing. Surprisingly, participants mentioned positive associations with the landing setup. 58\% (7 of 12) of the participants noticed the landing pad and mentioned that it helped them to understand the scenario: "I saw the square platform and it was clear what is going on." [P1]. Some participants mentioned they prefer the hook over the platform. They said the hook is an "optimal solution for the big drone" [P4], "a clever solution" [P9], and that they "would accept [the hook] rather than a platform" [P9].

6.5.2 Drone Localization. Auditive feedback played an important role for participants to localize the drone: "I tried to localize the drone according to the sound" [P8]. The sound is especially important if the drone is not visible: "I would need to trust the sound, when the drone is approaching from the back" [P2]. In addition to the rotor sounds, participants suggested additional audio feedback to signalize the landing: "Maybe a sound would have helped to signalize that the drone wants to land" [P7].

6.5.3 Proximity \& Control. Flying a drone near the head or shoulder was stated intimidating or as an injury risk by the participants: "[...] I fear that the drone gets stuck in my hair" [P5]. In contrast, landing a drone on the hand induced the feeling of being in control: "I want to see the drone. I think it is good to land on the hand because I want to be in control and be able to react easily." [P3]. Further, a controlled behavior was perceived positively: "I had feeling of sympathy because it was controlled. I did not feel threatened." [P6]

6.5.4 Drone Size. $75 \%$ (9 of 12) of the participants said that the small drone should be even smaller for at least one body location. Most participants wanted a smaller drone on the back (42\%), while $25 \%$ of participants said the drone should be smaller on other body locations. The large drone was mentioned as too big by $75 \%$ of participants. However, one participant also mentioned that drones should not be smaller than the small drone "since they would be difficult to notice" [P4]. One participant compared the small drone to "a small bird that lands on the hand" [P8]. This demonstrates more positive associations than the bigger drone, which was seen as "a bit threatening" [P6], "bulky" [P5], and "heavy" [P7].

6.5.5 Other Landing Locations. Beyond the implemented landing locations, participants suggested other landing setups. For example, "a kind of baby sling on the chest could make sense to keep the drone in the field of view" [P7] and "a big drone with an integrated hat could bring its own landing place" [P6]. A visible landing setup might also help to improve the bond between human and drone: "The backpack gives a guarantee that the drone will come back. I feel more connected to the drone" [P10].

6.5.6 Safety Risk and Trust. For the large drone participants stated that the injury risk is high when it approached from the back: "I feared that the drone would hurt me because of its size" [P6]. The participants stated that they had to trust the drone to land properly when they could not see it. "I could not see the drone, so I had to trust that the it lands safely" [P4]. 


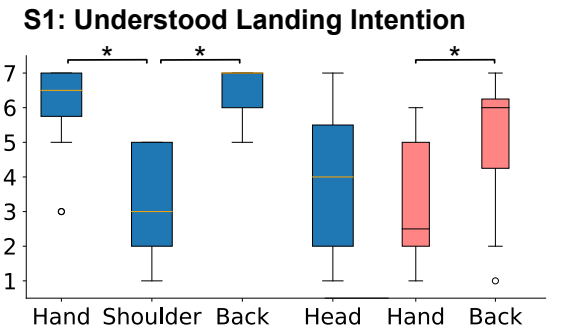

S4: Acceptable Landing Behavior

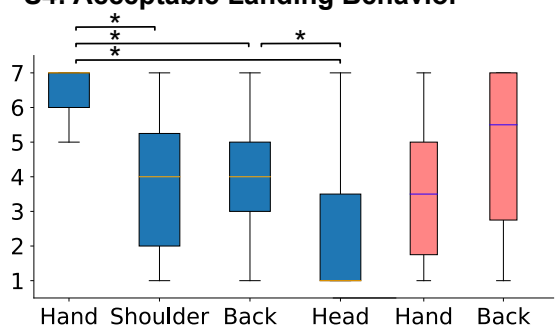

S7: Confident That Drone is Not Hurtful

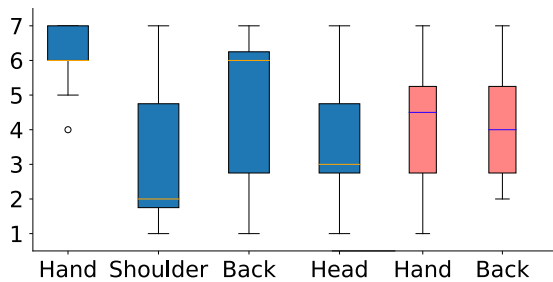

Small Drone
S2: Acceptable Visual Appearance

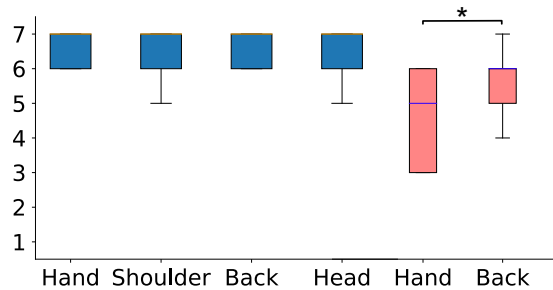

S5: Acceptable Drone Size

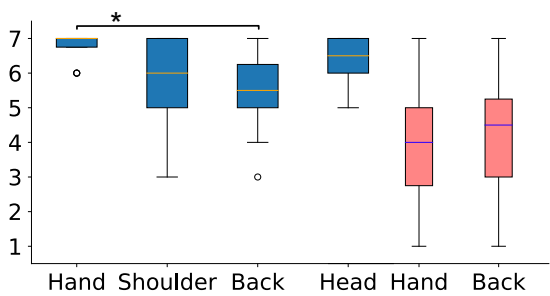

S8: Trusted Drone to Land Properly

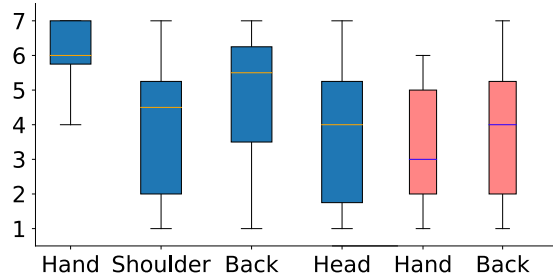

S3: Acceptable Noise

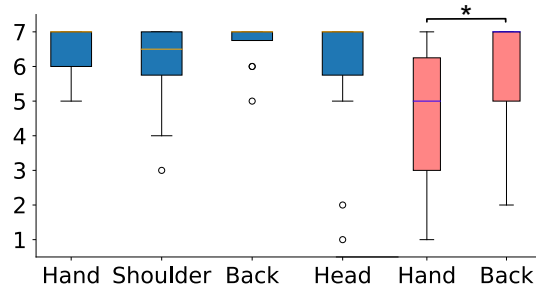

S6: Acceptable Landing Location

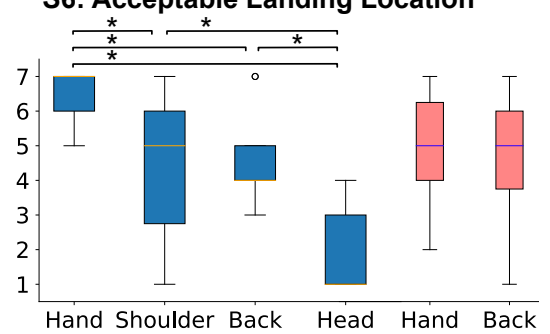

S9: Acceptable Approaching

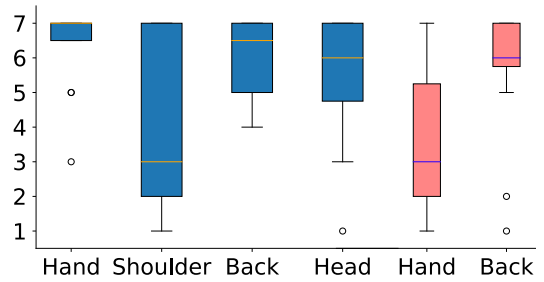

$\stackrel{*}{*} p<.05$

Figure 6: Participants 7-Point-Likert ratings of the nine statements.

6.5.7 Drone Approach. Positively rated approaches had flight paths that were perceived as "controlled", "steady", and "target-oriented". For low ratings, the most common reasons were that the drones were "too fast", "difficult to see", or "did not wait" for the user.

\section{COMBINED FINDINGS AND TAKEAWAYS}

This section compares the results from the online survey and VR study and discusses their similarities and differences.

\subsection{Which parts of the human body are suitable landing locations for drones? (RQ1)}

Through the VR study, we could confirm several findings from the online survey. We could confirm that the hands and the back can be suitable landing locations. This supports prior work on launching and catching drones using the hands [43], but also demonstrates that there are many more possibly acceptable locations for drone landings. In contrast, the shoulders, which were rated acceptable for landing in the online survey, were rated with a low acceptance by the participants of the VR study. Furthermore, we can confirm that the head is not well-suited for drone landing. For these landing locations, participants noted they would not accept the locations due to a high injury risk. This was made more apparent due to the increased immersion in the virtual environment compared to the online survey.

The acceptance in the online surveys was independent of the concrete landing platform. However, the VR study revealed that a good landing platform can help one to understand the drone's intention and can influence the acceptability of the overall system. Hence, the platforms should be carefully designed. While prior wearable design factors should be taken into account (e.g., [14, 17, 28, 53]), future work should investigate different landing mechanisms and their technical feasibility in more depth.

\subsection{How is landing acceptability influenced by the drone design? (RQ2)}

Despite the relatively neutral acceptance ratings in the MTurk, participants found the "traditional" quadcopter form factor highly acceptable when immersed in the VR scenario. This could be biased because participants only experienced two similar form-factors. However, both results indicate that there might not be a huge influence of visual appearance on the acceptability of on-body landing. This is particularly interesting, since prior work found that aesthetics play an important role for body-worn devices [39]. We 
believe that, despite the close proximity, our participants saw the drones as external objects rather than as a part of their bodies.

\subsection{How do activity and context influence drone landing acceptability? (RQ3)}

Through the online survey, we could gather first insights into the activity and context for which a user would accept drones landing on the body. We found that landing a drone would be considered more acceptable for work-related activities or in emergency rescue situations than, for example, for entertainment purposes. This highlights the idea that landing a drone on the human body could find application in more serious contexts in which critical tasks must be accomplished or lives must be saved.

\subsection{What influence do flight path and landing behavior have when landing on the human body? (RQ4)}

From the VR study, we learned that the participants wished for the drone to be in sight while landing. Further, a steady and controlled approaching procedure was required for acceptance of landing. Such a procedure should give the user time to prepare for the landing approach. Being in control of the situation and/or being able to react to unforeseen events was stated as a necessity for acceptance by the participants. For example, approaching the user from the back requires the user to trust in the system. Therefore, additional modalities, such as auditory feedback, might be of use. Also, the noise of the drone helped the participants to localize it when it was out of view. Together with auditory feedback that might further improve the way people localize drones and therefore increases trust in the landing procedure.

\subsection{General Findings Regarding VR Studies}

We further identified several interesting findings regarding the VR study. On the one hand, a real study with drones can be dangerous for the participants due to the high injury risk. Studying drones in VR comes with limited realism, but increases reproducibility [51]. Bringing the drone into the virtual world eliminates safety risks while still providing visual and auditory experiences in 3D space. On the other hand, some aspects are not covered by the virtual setting (e.g., haptics and airflow). One participant mentioned that VR was not convincing enough to feel immersed in the virtual setting: "It was not my hand. I knew that this was not really happening. I could abstract emotionally" [P1].

In addition, it is difficult to evaluate all aspects of drones (e.g., flight paths and noise) with an online survey. As the contradiction of the landing acceptance on the shoulder in the two studies shows, some aspects need to be investigated with real prototypes or closeto-real systems like VR. Here, we believe that in the online study the absence of motion and sound was leading to a higher landing acceptance on the shoulder. In contrast, the VR study revealed that the participants found landing on the shoulder less acceptable due to high injury risks and the proximity of the drone was found to be intimidating.

\section{LIMITATIONS \& FUTURE WORK}

In the following, we outline limitations of our research and suggest possible future research directions.

Environmental Conditions, Context and Activity. Drones are heavily influenced by environmental conditions such as the weather. Wind has a huge impact on the flying behavior of drones. This might impede proper landing maneuvers and thus might lower landing acceptability of users. This was not considered in our evaluation. Future research endeavors might investigate weather influence on drone landing capabilities and how users perceive possible associated risks, for example, through more realism-enhanced simulations that simulate events such as stormy weather conditions and shaky drones. Also, in our VR study, the participants could focus solely on the drone landing on them. In reality, this may differ depending on the context of a user. The acceptance of landing a drone on a user might be lower in specific social contexts such as being in a crowded place. Further, the task of a person might impact landing procedure and acceptability. For instance, rescue personnel that need an overview can benefit from drones autonomously starting from them and sending back information. However, when a person is carrying out life-saving measures (e.g., giving CPR), landing a drone on the hand is not acceptable. Therefore, context and activity play an important role for the acceptability of drone landing on the human body.

Studying Drones in Virtual Reality. In Wojciechowska et al.'s taxonomy on HDI research methodologies, VR studies achieve secondbest reality after co-located flights, resulting in medium realism and complexity while reducing safety risk [51]. This lower realism in VR is due to the lack of full sensory sensations, which are not possible with current VR systems. Because of the missing haptics, results with real drones might differ. However, VR still provides great detail when rendering video and audio. Future research might simulate appropriate haptics along with the VR experience; or, if risk can be eliminated, real specifically designed drones can be tested against our results. Our open source framework can be used to investigate such specifically designed drones for on-body landing.

Drone Design. Through the online survey, we obtained ratings on landing acceptability and suitability of differently designed drones. However, showing participants an image of a drone might not result in the same acceptability as experiencing the drone in reality. The proximity, flying speed, or sound could strongly affect acceptance. While our results uncovered initial insights into the acceptance, further landing acceptance factors (e.g., flight behavior, sound, and haptics) require further investigation. In addition, we acknowledge that these drones are the ones available on the market (see Figure 3). They are not designed to land on the body. Optimizing the design to land on the body might change the user's perception. Thereby, the drone design and the estimated body location for landing need to match. In the study, participants suggested different metaphors that can inform the design of the drones. This could help to improve users perception - particularly with regards to safety. For example, participants mentioned birds as a metaphor to create drones that are more accepted to land on the body (see Section 6.5). 
Further Research Directions. Our work presents an initial investigation into suitable locations for a drone to land on the body. We hope it will serve as a foundation for these additional research ideas, which go beyond the scope of this work. Future work should investigate further body locations, such as the front of the body and the legs, along with the long-term effects on the acceptability of on-body drone landings and the implications of real-world environmental conditions-such as wind, visibility, and social context-that arise from a particular user activity (e.g., climbing). Moreover, indepth studies must be conducted to fine-tune sound and motion behavior of the drones while approaching the human body for landing.

Safety and Perception of Safety. Safety is of utmost importance. In this work, we do not explore safety but we explore the perception of safety. Thus, our work is a first step towards including users' perception in the discussion on safety standards for human-drone interaction. With DJI hand-landing system ${ }^{4}$, landing drones on the human body has already started. Therefore, we argue that understanding preferences for drone landing on the human body is important and timely. As the technology is not fully developed yet and mostly limited to landing on the hand, it is important to understand which body parts seem acceptable for drone landing. This improves the discussion and future research on this subject. Especially as drones have the potential to bond with humans [16, $29,30]$ and become ubiquitous companions [7, 34] in the future. Creating such a bond between humans and drones through their interaction is studied actively in research [16, 29, 30, 34]. Future research could investigate further factors important to creating bonds like those of human to human interaction, where different social bonds are related to body regions [45]. On the technical side, there should be more research on the design of drones that promise a lower risk of injury (e.g., quadcopter with deformable propellers [38] or suitable emergency handling such as emergency stops which are already integrated in drone controllers. Future emergency stop mechanisms could consider human behavior in the loop).

\section{CONCLUSION}

This paper contributes a first understanding of drone landing on the human body. From two user studies, we derived location preferences and common themes for drone landing. Based on an online survey, we visualized the acceptance of different body locations while standing, sitting, walking, and climbing. Future drone designers can make use of these visualizations to find suitable body locations for their supported activities. We identified various common themes and appropriate landing locations from open-ended questions and the immersion into VR drone landing videos. Application designers that want to incorporate on-body drone landings should consider that different body locations influence perceived control over the drone and acceptability, that form factors can influence perceived landing suitability, and that contexts of a more serious nature might increase landing acceptability. We hope that this paper will stimulate future research on drones landing on, starting from, and being worn on the body.

\footnotetext{
${ }^{4}$ https://store.dji.com/product/mini-2
}

\section{REFERENCES}

[1] Parastoo Abtahi, Benoit Landry, Jackie (Junrui) Yang, Marco Pavone, Sean Follmer, and James A. Landay. 2019. Beyond The Force: Using Quadcopters to Appropriate Objects and the Environment for Haptics in Virtual Reality. In Proceedings of the 2019 CHI Conference on Human Factors in Computing Systems (Glasgow, Scotland Uk) (CHI '19). Association for Computing Machinery, New York, NY, USA, Article 359, 13 pages. https://doi.org/10.1145/3290605.3300589

[2] Parastoo Abtahi, David Y. Zhao, Jane L. E., and James A. Landay. 2017. Drone Near Me: Exploring Touch-Based Human-Drone Interaction. Proc. ACM Interact. Mob. Wearable Ubiquitous Technol. 1, 3, Article 34 (Sept. 2017), 8 pages. https: //doi.org/10.1145/3130899

[3] Jonas Auda, Martin Weigel, Jessica R. Cauchard, and Stefan Schneegaß. 2020. ProxyDrone: Autonomous Drone Landing on the Human Body. In iHDI@CHI. http://ceur-ws.org/Vol-2617/paper3.pdf

[4] Mauro Avila, Markus Funk, and Niels Henze. 2015. DroneNavigator: Using Drones for Navigating Visually Impaired Persons. In Proceedings of the 17th International ACM SIGACCESS Conference on Computers \& Accessibility (Lisbon, Portugal) (ASSETS '15). Association for Computing Machinery, New York, NY, USA, 327-328. https://doi.org/10.1145/2700648.2811362

[5] Sean Braley, Calvin Rubens, Timothy Merritt, and Roel Vertegaal. 2018. GridDrones: A Self-Levitating Physical Voxel Lattice for Interactive 3D Surface Deformations. In Proceedings of the 31st Annual ACM Symposium on User Interface Software and Technology (Berlin, Germany) (UIST '18). Association for Computing Machinery, New York, NY, USA, 87-98. https://doi.org/10.1145/3242587.3242658

[6] Jessica R. Cauchard, Jane L. E, Kevin Y. Zhai, and James A. Landay. 2015. Drone \& Me: An Exploration into Natural Human-Drone Interaction. In Proceedings of the 2015 ACM International foint Conference on Pervasive and Ubiquitous Computing (Osaka, Japan) (UbiComp '15). Association for Computing Machinery, New York, NY, USA, 361-365. https://doi.org/10.1145/2750858.2805823

[7] Jessica R. Cauchard, Mohamed Khamis, Jérémie Garcia, Matjaž Kljun, and Anke M. Brock. 2021. Toward a Roadmap for Human-Drone Interaction. Interactions 28, 2 (March 2021), 76-81. https://doi.org/10.1145/3447889

[8] Jessica R. Cauchard, Kevin Y. Zhai, Marco Spadafora, and James A. Landay. 2016. Emotion Encoding in Human-Drone Interaction. In The Eleventh ACM/IEEE International Conference on Human Robot Interaction (Christchurch, New Zealand) (HRI '16). IEEE Press, 263-270.

[9] Victoria Chang, Pramod Chundury, and Marshini Chetty. 2017. Spiders in the Sky: User Perceptions of Drones, Privacy, and Security. In Proceedings of the 2017 CHI Conference on Human Factors in Computing Systems (Denver, Colorado, USA) (CHI '17). Association for Computing Machinery, New York, NY, USA, 6765-6776. https://doi.org/10.1145/3025453.3025632

[10] Victoria Clarke, Virginia Braun, and Nikki Hayfield. 2015. Thematic analysis. Qualitative psychology: A practical guide to research methods (2015), 222-248.

[11] Artem Dementyev, Hsin-Liu (Cindy) Kao, Inrak Choi, Deborah Ajilo, Maggie Xu, Joseph A. Paradiso, Chris Schmandt, and Sean Follmer. 2016. Rovables: Miniature On-Body Robots as Mobile Wearables. In Proceedings of the 29th Annual Symposium on User Interface Software and Technology (Tokyo, Japan) (UIST '16). Association for Computing Machinery, New York, NY, USA, 111-120. https: //doi.org/10.1145/2984511.2984531

[12] Brittany A. Duncan and Robin R. Murphy. 2013. Comfortable approach distance with small Unmanned Aerial Vehicles. In 2013 IEEE RO-MAN. 786-792. https: //doi.org/10.1109/ROMAN.2013.6628409

[13] Brittany A. Duncan and Robin R. Murphy. 2017. Effects of Speed, Cyclicity, and Dimensionality on Distancing, Time, and Preference in Human-Aerial Vehicle Interactions. ACM Trans. Interact. Intell. Syst. 7, 3, Article 13 (Sept. 2017), 27 pages. https://doi.org/10.1145/2983927

[14] Lucy Dunne. 2008. Wearability in Wearable Computers. In Proceedings of the 2008 12th IEEE International Symposium on Wearable Computers (ISWC '08). IEEE Computer Society, USA, 125. https://doi.org/10.1109/ISWC.2008.4911605

[15] Jane L. E, Ilene L. E, James A. Landay, and Jessica R. Cauchard. 2017. Drone \& Wo: Cultural Influences on Human-Drone Interaction Techniques (CHI '17). Association for Computing Machinery, New York, NY, USA, 6794-6799. https: //doi.org/10.1145/3025453.3025755

[16] Sara Eriksson, Åsa Unander-Scharin, Vincent Trichon, Carl Unander-Scharin, Hedvig Kjellström, and Kristina Höök. 2019. Dancing With Drones: Crafting Novel Artistic Expressions Through Intercorporeality. In Proceedings of the 2019 CHI Conference on Human Factors in Computing Systems (Glasgow, Scotland Uk) (CHI '19). Association for Computing Machinery, New York, NY, USA, Article 617, 12 pages. https://doi.org/10.1145/3290605.3300847

[17] F. Gemperle, C. Kasabach, J. Stivoric, M. Bauer, and R. Martin. 1998. Design for wearability. In Digest of Papers. Second International Symposium on Wearable Computers (Cat. No.98EX215). 116-122.

[18] Michael A. Goodrich, Bryan S. Morse, Damon Gerhardt, Joseph L. Cooper, Morgan Quigley, Julie A. Adams, and Curtis Humphrey. 2008. Supporting wilderness search and rescue using a camera-equipped mini UAV. Fournal of Field Robotics 25, 1-2 (2008), 89-110. https://doi.org/10.1002/rob.20226 arXiv:https://onlinelibrary.wiley.com/doi/pdf/10.1002/rob.20226 
[19] Edward T. Hall. 1963. A system for the notation of proxemic behavior. American anthropologist 65, 5 (1963), 1003-1026.

[20] Chris Harrison and Haakon Faste. 2014. Implications of Location and Touch for On-Body Projected Interfaces. In Proceedings of the 2014 Conference on Designing Interactive Systems (Vancouver, BC, Canada) (DIS '14). Association for Computing Machinery, New York, NY, USA, 543-552. https://doi.org/10.1145/2598510. 2598587

[21] Hooman Hedayati, Ryo Suzuki, Daniel Leithinger, and Daniel Szafir. 2020. PufferBot: Actuated Expandable Structures for Aerial Robots. (2020). arXiv:2008.07615 [cs.RO]

[22] Matthias Hoppe, Pascal Knierim, Thomas Kosch, Markus Funk, Lauren Futami, Stefan Schneegass, Niels Henze, Albrecht Schmidt, and Tonja Machulla. 2018. VRHapticDrones: Providing Haptics in Virtual Reality through Quadcopters. In Proceedings of the 17th International Conference on Mobile and Ubiquitous Multimedia (Cairo, Egypt) (MUM 2018). Association for Computing Machinery, New York, NY, USA, 7-18. https://doi.org/10.1145/3282894.3282898

[23] Helge Huettenrauch, Kerstin S. Eklundh, Anders Green, and Elin A. Topp. 2006 Investigating Spatial Relationships in Human-Robot Interaction. In 2006 IEEE/RS International Conference on Intelligent Robots and Systems. 5052-5059. https: //doi.org/10.1109/IROS.2006.282535

[24] Kari Daniel Karjalainen, Anna Elisabeth Sofia Romell, Photchara Ratsamee, Asim Evren Yantac, Morten Fjeld, and Mohammad Obaid. 2017. Social Drone Companion for the Home Environment: A User-Centric Exploration. In Proceedings of the 5th International Conference on Human Agent Interaction (Bielefeld, Germany) (HAI '17). Association for Computing Machinery, New York, NY, USA 89-96. https://doi.org/10.1145/3125739.3125774

[25] Mohamed Khamis, Anna Kienle, Florian Alt, and Andreas Bulling. 2018. GazeDrone: Mobile Eye-Based Interaction in Public Space Without Augmenting the User. In Proceedings of the 4th ACM Workshop on Micro Aerial Vehicle Networks, Systems, and Applications (Munich, Germany) (DroNet'18). Association for Computing Machinery, New York, NY, USA, 66-71. https://doi.org/10.1145/3213526. 3213539

[26] Pascal Knierim, Thomas Kosch, Valentin Schwind, Markus Funk, Francisco Kiss, Stefan Schneegass, and Niels Henze. 2017. Tactile Drones - Providing Immersive Tactile Feedback in Virtual Reality through Quadcopters. In Proceedings of the 2017 CHI Conference Extended Abstracts on Human Factors in Computing Systems (Denver, Colorado, USA) (CHI EA '17). Association for Computing Machinery, New York, NY, USA, 433-436. https://doi.org/10.1145/3027063.3050426

[27] Pascal Knierim, Steffen Maurer, Katrin Wolf, and Markus Funk. 2018. QuadcopterProjected In-Situ Navigation Cues for Improved Location Awareness. In Proceedings of the 2018 CHI Conference on Human Factors in Computing Systems (Montreal QC, Canada) (CHI '18). Association for Computing Machinery, New York, NY, USA, 1-6. https://doi.org/10.1145/3173574.3174007

[28] James F. Knight, Daniel Deen-Williams, Theodoros N. Arvanitis, Chris Baber, Sofoklis Sotiriou, Stamatina Anastopoulou, and Michael Gargalakos. 2006. Assessing the Wearability of Wearable Computers. In 2006 10th IEEE International Symposium on Wearable Computers. 75-82. https://doi.org/10.1109/ISWC.2006.286347

[29] Joseph La Delfa, Mehmet Aydin Baytas, Rakesh Patibanda, Hazel Ngari, Rohit Ashok Khot, and Florian 'Floyd' Mueller. 2020. Drone Chi: Somaesthetic Human-Drone Interaction. In Proceedings of the 2020 CHI Conference on Human Factors in Computing Systems (Honolulu, HI, USA) (CHI '20). Association for Computing Machinery, New York, NY, USA, 1-13. https://doi.org/10.1145/3313831. 3376786

[30] Marc Lieser, Ulrich Schwanecke, and Jörg Berdux. 2021. Tactile Human-Quadrotor Interaction: MetroDrone. (2021). https://doi.org/10.1145/3430524.3440649

[31] Sven Mayer, Lars Lischke, and Paweł W. Wozniak. 2019. Drones for Search and Rescue. In International workshop on Human-Drone Interaction, CHI '19 Extended Abstracts (2019-05-04) (iHDI'19). Glasgow, Scotland, UK, 6. https://hal.archivesouvertes.fr/hal- 02128385

[32] Ross Mead and Maja J. Matarić. 2016. Perceptual Models of Human-Robot Proxemics. Springer International Publishing, Cham, 261-276. https://doi.org/10.1007/9783-319-23778-7 18

[33] Valiallah Mani Monajjemi, Jens Wawerla, Richard Vaughan, and Greg Mori. 2013 HRI in the sky: Creating and commanding teams of UAVs with a vision-mediated gestural interface. In 2013 IEEE/RSF International Conference on Intelligent Robots and Systems. 617-623. https://doi.org/10.1109/IROS.2013.6696415

[34] Florian Mueller and Matthew Muirhead. 2014. Understanding the Design of a Flying Jogging Companion. In Proceedings of the Adjunct Publication of the 27th Annual ACM Symposium on User Interface Software and Technology (Honolulu, Hawaii, USA) (UIST'14 Adjunct). Association for Computing Machinery, New York, NY, USA, 81-82. https://doi.org/10.1145/2658779.2658786

[35] Jonathan Mumm and Bilge Mutlu. 2011. Human-Robot Proxemics: Physical and Psychological Distancing in Human-Robot Interaction. In Proceedings of the 6th International Conference on Human-Robot Interaction (Lausanne, Switzerland) (HRI '11). Association for Computing Machinery, New York, NY, USA, 331-338 https://doi.org/10.1145/1957656.1957786

[36] Jawad Nagi, Alessandro Giusti, Gianni A. Di Caro, and Luca M. Gambardella. 2014. Human Control of UAVs Using Face Pose Estimates and Hand Gestures.
In Proceedings of the 2014 ACM/IEEE International Conference on Human-Robot Interaction (Bielefeld, Germany) (HRI '14). Association for Computing Machinery, New York, NY, USA, 252-253. https://doi.org/10.1145/2559636.2559833

[37] Wai Shan $\mathrm{Ng}$ and Ehud Sharlin. 2011. Collocated interaction with flying robots. In 2011 RO-MAN. 143-149. https://doi.org/10.1109/ROMAN.2011.6005280

[38] D. Q. Nguyen, G. Loianno, and V. A. Ho. 2020. Towards Design of a Deformable Propeller for Drone Safety. In 2020 3rd IEEE International Conference on Soft Robotics (RoboSoft). 464-469. https://doi.org/10.1109/RoboSoft48309.2020.9115983

[39] Matthew Pateman, Daniel Harrison, Paul Marshall, and Marta E. Cecchinato. 2018. The Role of Aesthetics and Design: Wearables in Situ. In Extended Abstracts of the 2018 CHI Conference on Human Factors in Computing Systems (Montreal QC, Canada) (CHI EA '18). Association for Computing Machinery, New York, NY, USA, 1-6. https://doi.org/10.1145/3170427.3188556

[40] G. Quiroz and S. J. Kim. 2017. A Confetti Drone: Exploring drone entertainment. In 2017 IEEE International Conference on Consumer Electronics (ICCE). 378-381. https://doi.org/10.1109/ICCE.2017.7889362

[41] Stefan Schneegass, Florian Alt, Jürgen Scheible, and Albrecht Schmidt. 2014. Midair Displays: Concept and First Experiences with Free-Floating Pervasive Displays. In Proceedings of The International Symposium on Pervasive Displays (Copenhagen, Denmark) (PerDis '14). Association for Computing Machinery, New York, NY, USA, 27-31. https://doi.org/10.1145/2611009.2611013

[42] Stefan Schneegass, Florian Alt, Jürgen Scheible, Albrecht Schmidt, and Haifeng Su. 2014. Midair Displays: Exploring the Concept of Free-Floating Public Displays. In CHI '14 Extended Abstracts on Human Factors in Computing Systems (Toronto, Ontario, Canada) (CHI EA '14). Association for Computing Machinery, New York, NY, USA, 2035-2040. https://doi.org/10.1145/2559206.2581190

[43] Jacob Self. 2019. Best Practices for Hand Launching and Catching a Drone - Let Us Drone. https://www.letusdrone.com/best-practices-for-hand-launchingand-catching-a-drone/

[44] Hazim Shakhatreh, Ahmad H. Sawalmeh, Ala Al-Fuqaha, Zuochao Dou, Eyad Almaita, Issa Khalil, Noor S. Othman, Abdallah Khreishah, and Mohsen Guizani. 2019. Unmanned Aerial Vehicles (UAVs): A Survey on Civil Applications and Key Research Challenges. IEEE Access 7 (2019), 48572-48634. https://doi.org/10.1109/ ACCESS.2019.2909530

[45] Juulia T. Suvilehto, E. Glerean, R. Dunbar, R. Hari, and L. Nummenmaa. 2015. Topography of social touching depends on emotional bonds between humans. Proceedings of the National Academy of Sciences 112 (2015), 13811 - 13816. https: //doi.org/10.1073/pnas.1519231112

[46] L. Takayama and C. Pantofaru. 2009. Influences on proxemic behaviors in humanrobot interaction. In 2009 IEEE/RS7 International Conference on Intelligent Robots and Systems. 5495-5502. https://doi.org/10.1109/IROS.2009.5354145

[47] Haodan Tan, Jangwon Lee, and Gege Gao. 2018. Human-Drone Interaction: Drone Delivery \& Services for Social Events. In Proceedings of the 2018 ACM Conference Companion Publication on Designing Interactive Systems (Hong Kong, China) (DIS '18 Companion). Association for Computing Machinery, New York, NY, USA, 183-187. https://doi.org/10.1145/3197391.3205433

[48] D. Tezza and M. Andujar. 2019. The State-of-the-Art of Human-Drone Interaction: A Survey. IEEE Access 7 (2019), 167438-167454. https://doi.org/10.1109/ACCESS. 2019.2953900

[49] M. L. Walters, K. Dautenhahn, S. N. Woods, K. L. Koay, R. Te Boekhorst, and D. Lee. 2006. Exploratory studies on social spaces between humans and a mechanicallooking robot. Connection Science 18, 4 (2006), 429-439. https://doi.org/10.1080/ 09540090600879513 arXiv:https://doi.org/10.1080/09540090600879513

[50] Anna Wojciechowska, Jeremy Frey, Esther Mandelblum, Yair AmichaiHamburger, and Jessica R. Cauchard. 2019. Designing Drones: Factors and Characteristics Influencing the Perception of Flying Robots. Proc. ACM Interact. Mob. Wearable Ubiquitous Technol. 3, 3, Article 111 (Sept. 2019), 19 pages. https://doi.org/10.1145/3351269

[51] Anna Wojciechowska, Jeremy Frey, Sarit Sass, Roy Shafir, and Jessica R. Cauchard. 2019. Collocated Human-Drone Interaction: Methodology and Approach Strategy. In 2019 14th ACM/IEEE International Conference on Human-Robot Interaction (HRI). 172-181. https://doi.org/10.1109/HRI.2019.8673127

[52] Alexander Yeh, Photchara Ratsamee, Kiyoshi Kiyokawa, Yuki Uranishi, Tomohiro Mashita, Haruo Takemura, Morten Fjeld, and Mohammad Obaid. 2017. Exploring Proxemics for Human-Drone Interaction. In Proceedings of the 5th International Conference on Human Agent Interaction (Bielefeld, Germany) (HAI '17). Association for Computing Machinery, New York, NY, USA, 81-88. https: //doi.org/10.1145/3125739.3125773

[53] Clint Zeagler. 2017. Where to Wear It: Functional, Technical, and Social Considerations in on-Body Location for Wearable Technology 20 Years of Designing for Wearability. In Proceedings of the 2017 ACM International Symposium on Wearable Computers (Maui, Hawaii) (ISWC '17). Association for Computing Machinery, New York, NY, USA, 150-157. https://doi.org/10.1145/3123021.3123042 